des opérations de vérification aux dispositions du protocole additionnel français. Il est important de noter que la présence de l'opérateur ou de son représentant est obligatoire pendant tout l'accès complémentaire.

Dès la réception du préavis, l'IRSN analyse la conformité de la demande d'accès complémentaire aux exigences du protocole additionnel français. Le CTE (ou l'IRSN, appui technique du CTE) en informe alors l'opérateur, afin notamment de déterminer l'ensemble des formalités à effectuer pour permettre l'entrée sur le site des différents intervenants ainsi que des équipements qui seront susceptibles d'être utilisés. Ce contact avec l'opérateur permet également de préciser les modalités d'accès dans l'installation, les consignes à suivre en termes notamment de protection des informations sensibles et l'organisation du travail.

Pour réaliser les opérations de vérification, les inspecteurs utilisent leurs propres équipements. L'emploi de ces matériels doit avoir été préalablement agréé par les autorités françaises.

À la fin des activités liées à l'accès complémentaire, le chef de l'équipe d'accompagnement dresse un procès-verbal relatant le résultat des opérations de vérification, qui est signé par les représentants de l'opérateur et le chef de l'équipe d'accompagnement. Les documents consultés par les inspecteurs sont inventoriés dans une annexe du procès-verbal.

\title{
3.4. Organisation française
}

\subsubsection{Cadre réglementaire}

Les missions des instances gouvernementales impliquées dans le domaine de la nonprolifération nucléaire sont précisées par décret. De plus, une documentation dédiée précise les règles relatives aux opérations d'importation et d'exportation de matières nucléaires.

\subsubsection{Instances gouvernementales}

Le Secrétariat général des affaires européennes (SGAE) a été créé en 2005 ; il succède au SGCI. Sous l'autorité du Premier ministre, le SGAE instruit et prépare les positions qui seront exprimées par la France au sein des institutions de l'Union européenne, qui comprend Euratom. Un comité d'experts apporte au SGAE l'appui technique nécessaire à l'exercice de ses attributions pour les questions relatives à l'application du traité instituant la CEEA (hormis le contrôle de sécurité). Ce comité d'experts est le Comité technique Euratom (CTE).

Le Comité technique Euratom (CTE), placé sous l'autorité du Premier ministre, est composé d'experts du Commissariat à l'énergie atomique et aux énergies alternatives. Il assure le suivi de la mise en œuvre des contrôles internationaux sur les matières nucléaires exercés en France par la Commission européenne au titre du chapitre VII du traité Euratom et par l'AIEA au titre de l'accord de garanties conclu entre la France, 
l'AIEA et Euratom. Il veille également à la mise en œuvre par la France du protocole additionnel à l'accord de garanties. Le CTE est également l'interlocuteur de la Commission européenne et de l'AIEA pour ces questions.

Par ailleurs, dans le cadre de la gestion patrimoniale des matières nucléaires nécessaires aux besoins de la défense, le CTE autorise les transferts de matières nucléaires entre les activités soumises au contrôle de sécurité Euratom et celles qui ne sont pas soumises à ce contrôle.

Le Commissariat à l'énergie atomique et aux énergies alternatives (CEA) conseille le gouvernement en matière de politique internationale dans le domaine nucléaire. Le CEA suit les évolutions scientifiques, techniques et économiques en vue d'éclairer le gouvernement, notamment dans la négociation des accords internationaux.

Le ministère des Affaires Étrangères a notamment pour missions de concevoir la politique extérieure, sous l'autorité du gouvernement, et de conduire et coordonner les relations internationales de la France. Pour ce qui concerne les aspects liés aux vérifications internationales dans le domaine nucléaire, le ministère s'appuie sur ses représentations permanentes auprès de l'Union européenne à Bruxelles, auprès de l'organisation des Nations unies à New-York et auprès des organisations internationales à Vienne (AIEA notamment).

Le gouverneur pour la France auprès de l'AIEA : diplomate du ministère des Affaires Étrangères et directeur des relations internationales du CEA, le gouverneur est en charge de l'ensemble du domaine de compétence de l'Agence et de l'application de certains accords multilatéraux. Il représente la France au Conseil des gouverneurs de l'AIEA.

La Direction générale de l'énergie et du climat (DGEC) du ministère en charge de l'Énergie, intervient dans la définition de la politique nucléaire française. La DGEC prépare notamment les positions françaises en vue des discussions internationales ou communautaires, et participe à la négociation des accords internationaux. Elle gère les déclarations des stocks de plutonium civil et des stocks d'uranium hautement enrichi.

Autres institutions : d'autres ministères, notamment le ministère de la Défense, interviennent dans le contrôle des matières nucléaires, plus particulièrement de celles affectées aux besoins de la défense.

\subsubsection{Institut de radioprotection et de sûreté nucléaire}

L'IRSN apporte son soutien technique à la fois aux pouvoirs publics et aux opérateurs pour la mise en œuvre, sur le territoire national, des contrôles internationaux dans le domaine de la non-prolifération. L'IRSN, support technique des autorités gouvernementales, a en effet un positionnement particulier dans ce domaine dans la mesure où il conseille les opérateurs aussi bien dans la démarche de déclaration que dans le processus d'inspection. Une convention signée par l'IRSN avec le CEA et le CTE précise les missions confiées à l'Institut dans le domaine du contrôle des matières nucléaires, à savoir : 
- la gestion des déclarations françaises prévues par les traités et accords ;

- la préparation, l'accompagnement et le suivi des inspections internationales. Dans ce cadre, l'IRSN doit à la fois veiller au respect des engagements internationaux de la France auprès des organismes concernés (AIEA et Euratom) et protéger les intérêts des opérateurs français (protection des informations sensibles, des secrets industriels...) ;

- l'analyse de la documentation technique due par les assujettis aux organismes internationaux de contrôle ainsi que l'assistance et le conseil aux assujettis dans le cadre de l'application des traités et accords ;

- l'analyse et le suivi des évolutions ou projets d'évolution du cadre juridique national et international ;

- la formation des industriels français ;

- la participation à certains groupes de travail internationaux.

\section{\#FOCUS}

\section{L'IRSN, appui des opérateurs face aux organismes internationaux en charge du contrôle de non-prolifération}

En adhérant aux deux principaux régimes de non-prolifération, relatifs l'un aux armes nucléaires, l'autre aux armes chimiques (chapitre 4), la France a pris des engagements forts à l'égard de la communauté internationale et des organisations chargées de la mise en œuvre de ces traités (Agence internationale de l'énergie atomique - AIEA - et Organisation pour l'interdiction des armes chimiques - OIAC). De plus, l'appartenance de la France à l'Union européenne implique également un contrôle des industriels du nucléaire par Euratom.

Ces engagements conduisent notamment à élaborer des déclarations et à recevoir des inspections de ces organisations sur le territoire national.

Par leurs activités de contrôle, les inspecteurs de l'OIAC, de l'AIEA et d'Euratom pourraient avoir accès à des informations sensibles du point de vue :

- du secret commercial, par l'accès à des listes de produits, de clients, de tonnages de matières ;

- de la propriété industrielle, par l'accès à des plans, procédés industriels, formules chimiques ;

- de la non-prolifération, pour l'usine Georges Besse II, l'accès à certaines zones pouvant révéler des informations dont la diffusion pourrait conduire à une dissémination de la technologie d'enrichissement par centrifugation;

- de la défense nationale, par l'accès à des installations qui, outre des activités industrielles ou de recherches civiles, conduisent également des activités pour le compte de la défense nationale. 
En tant qu'appui technique des autorités françaises, l'IRSN a notamment pour rôle, lors de l'accompagnement des inspections internationales en France, de défendre les intérêts nationaux. À cet égard, l'Institut veille à limiter l'accès des inspecteurs aux informations aux seules exigences requises au titre d'un traité, d'un accord ou d'une convention internationale ou multilatérale; ou à trouver des solutions de contournement pour permettre aux inspecteurs de s'acquitter de leur tâche sans pour autant dévoiler des informations jugées confidentielles par les industriels.

Par voie de conséquence, l'IRSN se trouve ainsi régulièrement en posture de soutien ou de conseil à l'opérateur. Faisant ainsi l'interface avec les inspecteurs, l'IRSN conseille l'opérateur sur l'interprétation et la mise en œuvre des textes applicables, lui signalant par exemple les cas où les inspecteurs pourraient sortir de leurs prérogatives ou lorsque leurs interrogations sont justifiées mais formulées de façon inadéquate (en termes de méthodes plutôt que d'objectifs). L'IRSN s'efforce en tout état de cause de faciliter la relation entre l'opérateur et les inspecteurs tout en recentrant ceux-ci sur les limites de leur mandat ou sur ses objectifs, en proposant par exemple d'autres solutions que celles initialement envisagées.

Cette posture de conseil et de support des industriels français dans les domaines chimique et nucléaire dans le contexte des inspections internationales de nonprolifération constitue une originalité certaine au sein des missions de l'IRSN, traditionnel support technique des autorités. 
